Yunqiao Dong et al., Int. J. Comp. Meth. and Exp. Meas., Vol. 4, No. 2 (2016) 80-89

\title{
A NOVEL SINGULAR ELEMENT FOR EVALUATING 3D STRESS INTENSITY FACTOR OF THE THROUGH-THICKNESS CRACK
}

\author{
YUNQIAO DONG ${ }^{1}$, JIANMING ZHANG ${ }^{1}$, LEI HAN ${ }^{1}$, GUIZHONG XIE ${ }^{2}$ \& RUI HE ${ }^{1}$ \\ ${ }^{1}$ State Key Laboratory of Advanced Design and Manufacturing for Vehicle Body, Hunan University, \\ Changsha 410082, China. \\ ${ }^{2}$ Mechanical and Electrical Engineering Institute, Zhengzhou University of Light Industry, \\ Zhengzhou 450002, China.
}

\begin{abstract}
A novel singular element is presented to evaluate the stress intensity factor (SIF) of the through-thickness crack in this paper. The new element takes into account the special variation of the displacements around the intersection of the crack front and the free surface. The intersection between the crack front and the free surface is named singular point. The proposed element has a vertex which coincides with the singular point. Accurately capturing the distribution of displacements in the vicinity of the singular point is of crucial importance in the implementation of dual boundary element method (DBEM) for the through-thickness crack problems. The element with usual shape functions doesn't lead to accurate solutions unless extremely fine meshes are used. With these new singular elements, more accurate results for the displacement filed around the singular point and the SIF can be obtained. Numerical examples have demonstrated the accuracy and efficiency of the proposed method.

Keywords: dual boundary element method, stress intensity factor, through-thickness crack, vertex singularity.
\end{abstract}

\section{INTRODUCTION}

The through-thickness crack problem widely appears in engineering problem. Accurate evaluation of stress intensity factor (SIF) is of great importance for this problem. Many numerical methods have been proposed to evaluate SIF such as the finite element method (FEM), the extended finite element method (XFEM) and the boundary element method (BEM). Compared to FEM and XFEM, the BEM seems to be more attractive for its dimension reduction feature [1-2]. However, the conventional BEM cannot apply to crack problems directly, because a singular system of equations is always obtained for the coincidence of the crack boundaries. To overcome this difficulty, various methods within the scope of BEM have been presented, for instance the special Green's method [3], the multi-domain techniques [4], the displacement discontinuity or dislocation method [5], the Galerkin symmetric method [6], a direct traction boundary integral equation method [7] and the dual boundary element method (DBEM) [8-12]. Among the above methods, the DBEM is a more promising method.

The displacements around the crack front have square root variation of $r, r$ being the distance to the crack front. The element with the usual shape functions which allow for polynomial variation only is not suitable for modelling crack front regions. Many researchers have proposed special crack tip element, including quarter-point elements [13] and mid-side node elements where special shape functions are introduced [14]. 
The intersection between the crack front and the free surface is named singular point. Little literature considers the special variation of the displacement filed around the singular point on the free surface, although the vertex singularity exists in the through-thickness crack problem [15-17]. Therefore, a novel singular element is proposed in the paper.

The new singular element has a vertex which coincides with the singular point. These elements are collocated on the free surfaces which include the singular points rather than on crack surfaces. The vertex singularity is affected by many factors, such as the Poisson's ratio, the thickness of the crack and so on [15-19]. But from the results in these literature, it can be seen that the vertex singularity approach square root of $r$ in most cases. For simplicity, we design a new element which has square root variation to model the special displacement filed around the singular point. Numerical examples are presented to verify that our method is suitable for solving the through-thickness crack problems with different Poisson's ratio.

This paper is organized as follows. In section 2, the DBEM are described. Section 3 introduces the vertex singularity. The new singular element is presented in Section 4. Numerical examples are given in Section 5. This paper ends with conclusions in Section 6.

\section{THE DUAL BOUNDARY ELEMENT METHOD}

Consider a cracked body as shown in Fig. 1, with $\Gamma^{+}$and $\Gamma^{-}$referring to the lower and upper crack surfaces, respectively, and $S$ to the rest of the boundary. The displacement boundary integral equation in absence of body forces can be written as

$$
c_{i j}\left(P_{S}\right) u_{j}\left(P_{S}\right)=\int_{S+\Gamma^{+}+\Gamma^{-}} u_{i j}^{*}\left(P_{S}, Q\right) t_{j}(Q) d \Gamma(Q)-\int_{S+\Gamma^{+}+\Gamma^{-}} t_{i j}^{*}\left(P_{S}, Q\right) u_{j}(Q) d \Gamma(Q)
$$

where $P_{s}$ and $Q$ are the source point and the field point, respectively. $c_{i j}$ is a function of the geometry variation at the boundary point. $u_{j}$ and $t_{j}$ represent the components of displacement and traction. $u_{i j}^{*}$ and $t_{i j}^{*}$ are the Kelvin fundamental solutions for displacement and traction. The Kelvin fundamental solutions $u_{i j}^{*}$ and $t_{i j}^{*}$ are given by:

$$
u_{i j}^{*}=\frac{1}{16 \pi G(1-v) r}\left[(3-4 v) \delta_{i j}+r_{, i} r_{, j}\right]
$$

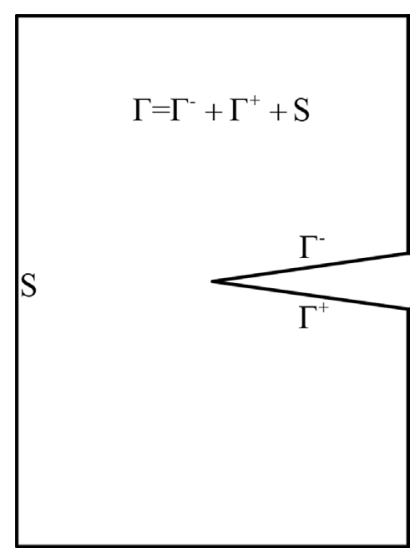

Figure 1: Cracked body. 


$$
t_{i j}^{*}=-\frac{1}{8 \pi(1-v) r^{2}}\left\{\frac{\partial r}{\partial n}\left[(1-2 v) \delta_{i j}+3 r_{, i} r_{, j}\right]-(1-2 v)\left(r_{, i} n_{j}-r_{, j} n_{, i}\right)\right\}
$$

where $r$ is the distance between the source point $P_{s}$ and the field point $Q ; G, v$ and $\delta_{i j}$ represent the shear modulus, the Poisson's ratio and the Kronecker delta, respectively; $n$ denotes the unit outward normal vector at the point $Q$ on the boundary. $n_{i}$ and $n_{j}$ are the components of the normal $n$, and $r_{, i}=\partial r / \partial x_{i}, r_{, j}=\partial r / \partial x_{j}$.

The traction boundary integral equation in absence of body forces can be written as

$$
\frac{1}{2} t_{j}\left(P_{S}\right)=n_{i}\left(P_{S}\right) \int_{S+\Gamma^{+}+\Gamma^{-}} U_{i j k}^{*}\left(P_{S}, Q\right) t_{k}(Q) d \Gamma(Q)-n_{i}\left(P_{S}\right) \int_{S+\Gamma^{+}+\Gamma^{-}} T_{i j k}^{*}\left(P_{S}, Q\right) u_{k}(Q) d \Gamma(Q)
$$

where $n_{i}\left(P_{S}\right)$ denotes one component of the unit outward normal vector at the point $P_{s}$ on the boundary. $U_{i j k}^{*}$ and $T_{i j k}^{*}$ contain several derivatives of the Kelvin fundamental solutions together with elastic constants. Expression for $U_{i j k}^{*}$ and $T_{i j k}^{*}$ are:

$$
\begin{gathered}
U_{i j k}^{*}\left(P_{S}, Q\right)=\frac{3 r_{, i} r_{, j} r_{, k}+(1-2 \nu)\left(r_{, i} \delta_{j k}+r_{, j} \delta_{i k}-r_{, k} \delta_{i j}\right)}{8 \pi(1-\nu) r^{2}} \\
T_{i j k}^{*}\left(P_{S}, Q\right)=\frac{G}{4 \pi(1-v) r^{3}}\left\{(1-2 \nu)\left(\delta_{i k} n_{j}+\delta_{k j} n_{i}+3 r_{, j} r_{, i} n_{k}\right)-(1-4 v) \delta_{i j} n_{k}\right. \\
\left.+3 v r_{, k}\left(r_{, i} n_{j}+r_{, j} n_{i}\right)+3 \frac{\partial r}{\partial n}\left[(1-2 v) \delta_{i j} r_{, k}+\nu\left(\delta_{i k} r_{, j}+\delta_{j k} r_{, i}\right)-5 r_{, i} r_{, k} r_{, j}\right]\right\}
\end{gathered}
$$

Traction equilibrium $\left.t_{k}\left(P_{S}\right)\right|_{\Gamma^{+}}=\left.t_{k}\left(P_{S}\right)\right|_{\Gamma^{-}}$is assumed on the crack faces. The kernels $u_{i j}^{*}$, $t_{i j}^{*}, U_{i j k}^{*}$ and $T_{i j k}^{*}$ have following properties:

$$
\begin{gathered}
\left.u_{i j}^{*}\left(P_{S}, Q\right)\right|_{\Gamma^{+}}=\left.u_{i j}^{*}\left(P_{S}, Q\right)\right|_{\Gamma^{-}},\left.\quad t_{i j}^{*}\left(P_{S}, Q\right)\right|_{\Gamma^{+}}=-\left.t_{i j}^{*}\left(P_{S}, Q\right)\right|_{\Gamma^{-}} \\
\left.U_{i j k}^{*}\left(P_{S}, Q\right)\right|_{\Gamma^{+}}=\left.U_{i j k}^{*}\left(P_{S}, Q\right)\right|_{\Gamma^{-}},\left.\quad T_{i j k}^{*}\left(P_{S}, Q\right)\right|_{\Gamma^{+}}=-\left.T_{i j k}^{*}\left(P_{S}, Q\right)\right|_{\Gamma^{-}} .
\end{gathered}
$$

Thus a new pair of boundary integral equations can be obtained as follows:

$$
\begin{gathered}
c_{i j}\left(P_{S}\right) u_{j}\left(P_{S}\right)=\int_{S} u_{i j}^{*}\left(P_{S}, Q\right) t_{j}(Q) d \Gamma-\int_{S} t_{i j}^{*}\left(P_{S}, Q\right) u_{j}(Q) d \Gamma \\
-\int_{\Gamma^{+}} t_{i j}^{*}\left(P_{S}, Q^{+}\right) \Delta u_{j}(Q) d \Gamma \\
t_{j}\left(\left.P_{S}\right|_{\Gamma^{+}}\right)=n_{i}\left(\left.P_{S}\right|_{\Gamma^{+}}\right) \int_{S} U_{i j k}^{*}\left(\left.P_{S}\right|_{\Gamma^{+}}, Q\right) t_{k}(Q) d \Gamma \\
-n_{i}\left(\left.P_{S}\right|_{\Gamma^{+}}\right) \int_{S} T_{i j k}^{*}\left(\left.P_{S}\right|_{\Gamma^{+}}, Q\right) u_{k}(Q) d \Gamma-n_{i}\left(\left.P_{S}\right|_{\Gamma^{+}}\right) \int_{\Gamma^{+}} T_{i j k}^{*}\left(\left.P_{S}\right|_{\Gamma^{+}}, Q\right) \Delta u_{k}(Q) d \Gamma
\end{gathered}
$$

where $\Delta u_{k}(Q)$ represents the open displacement on the crack surface, $u_{j}(Q)$ and $t_{j}(Q)$ represent the displacement and traction on the uncracked surfaces. Equation (7a) is collocated on the uncracked boundary and Eqn (7b) is used on the lower crack surface.

It should be noted that eqn (7a) and (b) can also be employed for the problems containing multiple crack surfaces in a finite space. For the problems containing multiple crack surfaces 
in an infinite space, only eqn (7b) is required. This formulation has the advantage of a smaller system of equations than the conventional DBEM since only one of the crack surface needs to be discretized.

\section{THE VERTEX SINGULARITY}

In this section, we introduce the vertex singularity at the singular point (intersection of the crack front and the free surface). Benthem (1977) [15], by using three-dimensional separation of variables for a quarter infinite crack in a half space, showed that a vertex singularity exists at the singular point. Then a lot of literature [16-19] has been presented to discuss the singularity order at the singular point, including analytic method and numerical method. The results from these papers show that the vertex singularity is close to square root of $r$ in most cases. The following example can also illustrate this point.

The displacements of a 3D through-thickness crack are simulated by finite element software ABAQUS with 2.44 million elements. The displacement U2 around the crack front for different cross section along the thickness direction is shown in Fig. 2. The numbers $0-1.5$ represent the distance of different cross section to the free surface. 0 denotes the free surface, and 1.5 is the middle cross section of the cracked body along the thickness direction.

The displacements of the middle cross section in the vicinity of the crack front have square root variation of $r$. It can be seen from Fig. 2 that the displacements $\mathrm{U} 2$ of different cross section including the free surface have almost the same variation trend. This means that the displacements around the singular point on the free surface have similar square root variation.

\section{THE NEW SINGULAR ELEMENT}

From the previous section, it can be seen that the displacements around the singular point on the free surface have similar square root variation of $r$. But the accurate variation of the displacement field depends on the order of the vertex singularity. For simplicity, a new singular element which has square root variation is presented to model the special displacement filed around the singular point in this section.

The new singular elements have a vertex which coincides with the singular point. These elements are collocated on the free surface rather than on crack surface as shown in Fig. 3.

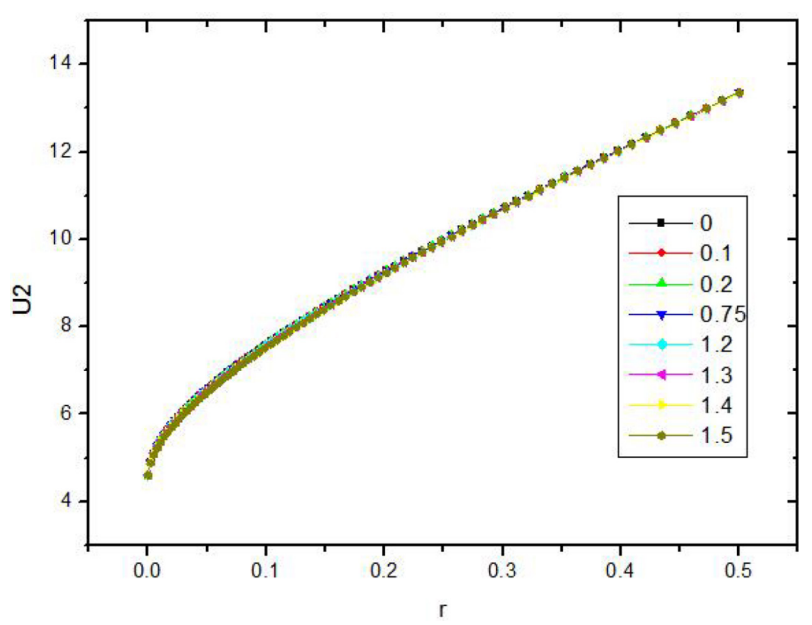

Figure 2: Variation of $\mathrm{U} 2$ with $r$ for different cross section along the thickness direction. 


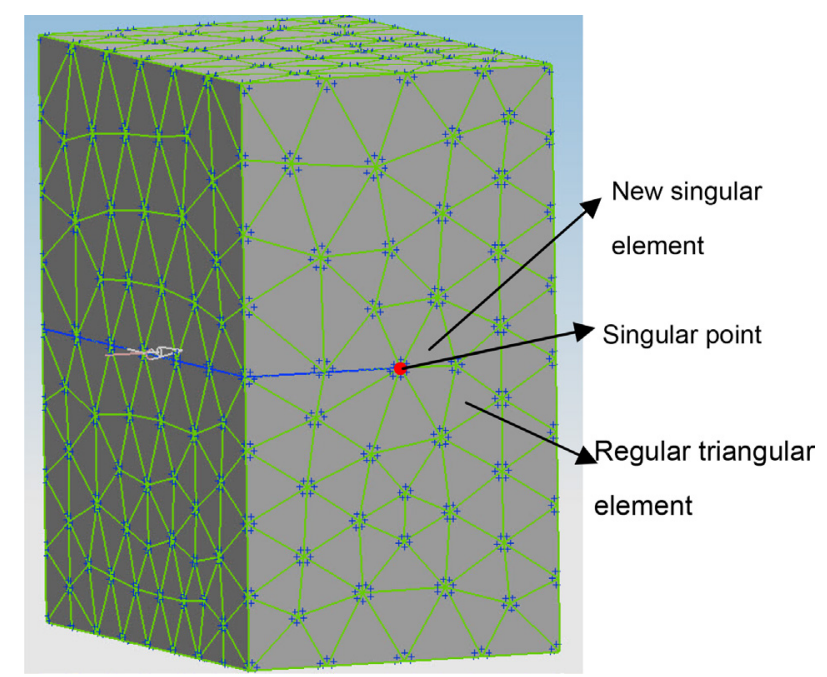

Figure 3: The position of the new singular element.

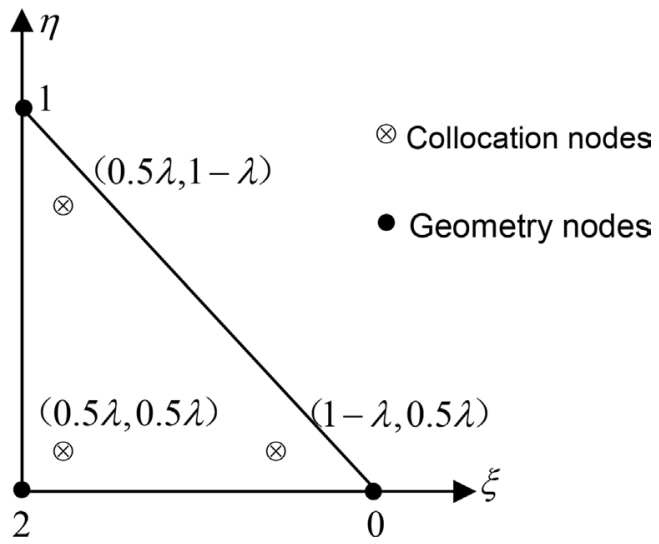

Figure 4: The new triangular element.

Let us assume that the singular point lies on node 2 of the new triangular element shown in Fig. 4. In order to get the desired square root variation, the shape functions of the new element should be of the following form:

$$
N^{i}=a_{0}^{i}+\frac{a_{1}^{i} \xi}{\sqrt{\xi+\eta}}+\frac{a_{2}^{i} \eta}{\sqrt{\xi+\eta}}, i=0,1,2 .
$$

The shape functions in eqn (8) must satisfy the conditions:

$$
N^{i}\left(\xi_{j}, \eta_{j}\right)=\delta_{i j}, i, j=0,1,2 .
$$


where $\delta_{i j}$ is the Kronecker delta, and $\left(\xi_{i}, \eta_{i}\right)$ are the functional coordinates for the discontinuous element in the $(\xi, \eta)$ coordinate system, as illustrated in Fig. 4. Using eqn (9) for each $i$ in eqn (8), a set of $3 \times 3$ linear system of equations is obtained. Solving this system of equations will yield the coefficients $a_{j}^{i}$. Assuming $\lambda=0.25$, the shape functions for the new singular element are obtained as:

$$
\begin{aligned}
& N^{0}(\xi, \eta)=-0.574165738+1.896662954 \frac{\xi}{\sqrt{\xi+\eta}}+0.4 \frac{\eta}{\sqrt{\xi+\eta}} \\
& N^{1}(\xi, \eta)=-0.574165738+0.4 \frac{\xi}{\sqrt{\xi+\eta}}+1.896662954 \frac{\eta}{\sqrt{\xi+\eta}} \\
& N^{2}(\xi, \eta)=2.148331476-2.296662954 \frac{\xi}{\sqrt{\xi+\eta}}-2.296662954 \frac{\eta}{\sqrt{\xi+\eta}}
\end{aligned}
$$

\section{NUMERICAL EXAMPLES}

To verify the accuracy and efficiency of the proposed singular element, several examples are presented in this section. The SIFs are evaluated by the following expressions:

$$
\begin{aligned}
& K_{I}=\frac{E}{4\left(1-v^{2}\right)} \sqrt{\frac{\pi}{2 r}} \Delta u_{b} \\
& K_{I I}=\frac{E}{4\left(1-v^{2}\right)} \sqrt{\frac{\pi}{2 r}} \Delta u_{n} \\
& K_{I I I}=\frac{E}{4(1+v)} \sqrt{\frac{\pi}{2 r}} \Delta u_{t}
\end{aligned}
$$

where $E$ is Young's modulus and $v$ represents Poisson's ratio. $\Delta u_{b}, \Delta u_{n}, \Delta u_{t}$ are projections of the crack open displacements on the local coordinate directions (normal, binormal and tangential) at the crack front as shown in Fig. 5.

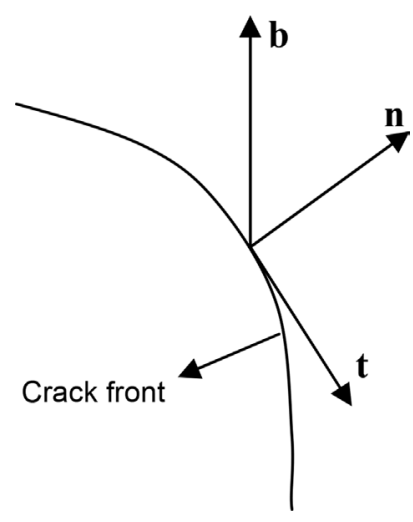

Figure 5: Local coordinate system at the crack front. 


\subsection{Example 1}

In the first example, we concern a through-thickness crack bar specimen of thickness $t$, width $w$ and total height $2 h$, with a crack length a through the thickness as shown in Fig. 6 . The ends of specimen are subjected to a uniform uniaxial tensile stress $\sigma$ in the y-direction, perpendicular to the crack. The meshes are shown in Fig. 7. The normalized SIFs, (i.e. $K_{I} / \sigma \sqrt{\pi a}$ ) for

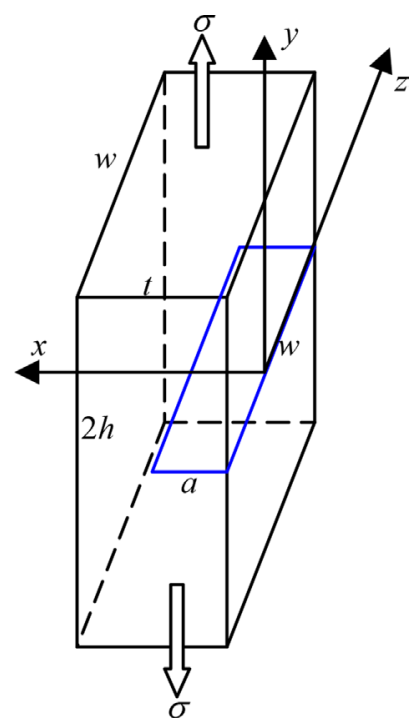

Figure 6: Geometry model of a through-thickness crack.

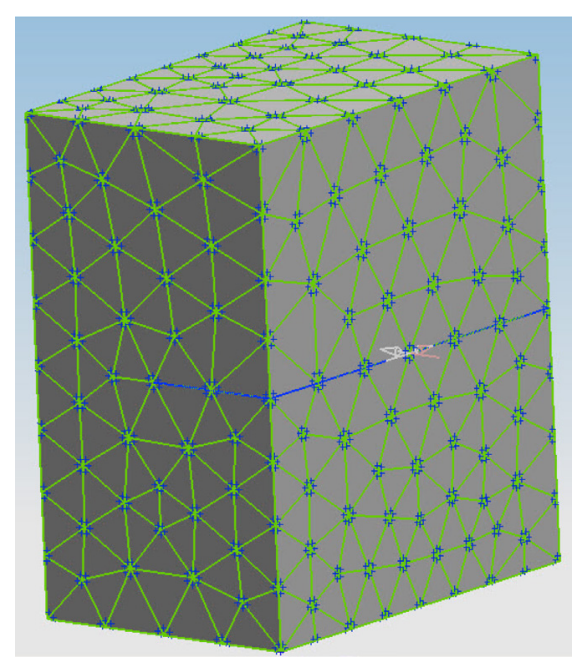

Figure 7: Mesh model of a through-thickness crack. 


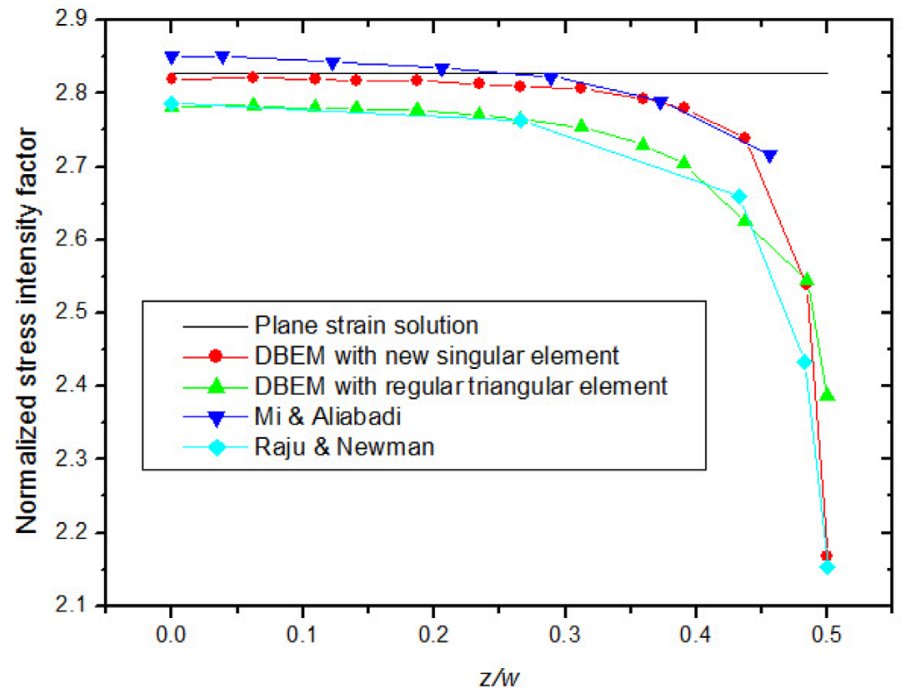

Figure 8: Normalized SIFs along the crack front.

Table 1: Normalized SIFs at the center of the bar with different Poisson's ratios.

\begin{tabular}{lllllll}
\hline$v$ & 0.1 & 0.15 & 0.2 & 0.25 & 0.3 & 0.35 \\
$\mathrm{~K}_{\mathrm{I}}$ & 2.78054 & 2.78868 & 2.80190 & 2.82019 & 2.8437 & 2.8727 \\
\hline
\end{tabular}

$t / a=2, w / a=3, h / a=1.75$ along the crack front are illustrated in Fig. 8 together with the results obtained by Raju \& Newman [20] and Mi \& Aliabadi [10]. 'DBEM with regular triangular element' represents that regular triangular elements are used around the singular point. 'DBEM with new singular element' denotes our proposed method. The SIF at the center of the bar evaluated by the proposed method is 2.82019 . This value is within $0.24 \%$ of the plane strain solution in Ref. [21].

\subsection{Example 2}

The vertex singularity depends on the value of Poisson's ratio. In order to verify the versatility of the proposed singular element, a series of Poisson's ratios are presented. The problem considered in this example is the same as that in example 1. The SIFs evaluated at the center of the bar with different Poisson's ratio are shown in Table 1. $v$ is the Poisson's ratio and $\mathrm{K}_{\mathrm{I}}$ is the normalized SIF. It can be seen from Table 1 that accurate results can be obtained for different Poisson's ratio.

\section{CONCLUSIONS}

A new singular element with square root variation is proposed to analyze the throughthickness crack problem in this paper. The new element takes into account the special variation of the displacements in the neighborhood of the singular point. The proposed 
element has a vertex which coincides with the singular point, and these elements are collocated on the free surfaces rather than on crack surfaces. With the new singular elements, the special behavior of displacements around the singular point can be captured accurately. Numerical examples showed that more accurate SIF can be obtained by our proposed method within $0.24 \%$ of the plane strain solution.

\section{ACKNOWLEDGEMENTS}

This work was supported in part by National Science Foundation of China under grant number 11472102, in part by Open Research Fund of Key Laboratory of High Performance Complex Manufacturing, Central South University under grant number Kfkt2013-05, and in part by State Key Laboratory of Advanced Design and Manufacturing for Vehicle Body under grant number 71375003.

\section{REFERENCES}

[1] Yan, A.M. \& Nguyen-Dang, H., Multiple-cracked fatigue crack growth by BEM. Computational Mechanics, 16(5), pp. 273-280, 1995. http://dx.doi.org/10.1007/BF00350716

[2] Liu, Y.J. \& Xu, N., Modeling of interface cracks in fiber-reinforced composites with the presence of interphases using the boundary element method. Mechanics of Materials, 32(12), pp. 769-783, 2000. http://dx.doi.org/10.1016/S0167-6636(00)00045-4

[3] Telles, J.C.F., Castor, G.S. \& Guimaraes, S., A numerical Green's function approach for boundary elements applied to fracture mechanics. International Journal for Numerical Methods in Engineering, 38(19), pp. 3259-3274, 1995. http://dx.doi.org/10.1002/nme.1620381906

[4] Blandford, G.E., Anthony, R.I. \& James, A.L., Two-dimensional stress intensity factor computations using the boundary element method. International Journal for Numerical Methods in Engineering, 17(3), pp. 387-404, 1981. http://dx.doi.org/10.1002/nme.1620170308

[5] Crouch, S.L., Antony, M.S. \& Rizzo, F.J., Boundary element methods in solid mechanics. Journal of Applied Mechanics, 50, p. 704, 1983.

http://dx.doi.org/10.1115/1.3167130

[6] Sirtori, S., Maier, G., Novati, G. \& Miccoli, S., A Galerkin symmetric boundaryelement method in elasticity: formulation and implementation. International Journal for Numerical Methods in Engineering, 35(2), pp. 255-282, 1992. http://dx.doi.org/10.1002/nme.1620350204

[7] Xie, G., Zhang, J., Huang, C., Lu, C. \& Li, G., A direct traction boundary integral equation method for three-dimension crack problems in infinite and finite domains. Computational Mechanics, 53(4), pp. 575-586, 2014. http://dx.doi.org/10.1007/s00466-013-0918-8

[8] Hong, H.K. \& Chen, J.T., Derivations of integral equations of elasticity. Journal of Engineering Mechanics, 114(6), pp. 1028-1044, 1988. http://dx.doi.org/10.1061/(ASCE)0733-9399(1988)114:6(1028)

[9] Chen, J.T. \& Hong, H.K., Review of dual boundary element methods with emphasis on hyprsingular integrals and divergent series. Applied Mechanics Reviews, 52, pp. 17-33, 1999.

http://dx.doi.org/10.1115/1.3098922 
[10] Mi, Y. \& Aliabadi, M.H., Dual boundary element method for three-dimensional fracture mechanics analysis. Engineering Analysis with Boundary Elements, 10(2), pp. 161-171, 1992.

http://dx.doi.org/10.1016/0955-7997(92)90047-B

[11] Pan, E. \& Yuan, F.G., Boundary element analysis of three-dimensional cracks in anisotropic solids. International Journal for Numerical Methods in Engineering, 48(2), pp. 211-237, 2000.

http://dx.doi.org/10.1002/(SICI)1097-0207(20000520)48:2\%3C211::AID-

NME875\%3E3.0.CO;2-A

[12] Chen, W.H. \& Chen, T.C., An efficient dual boundary element technique for a two-dimensional fracture problem with multiple cracks. International Journal for Numerical Methods in Engineering, 38(10), pp. 1739-1756, 1995.

http://dx.doi.org/10.1002/nme.1620381009

[13] Ariza, M.P., Saez, A. \& Dominguez, J., A singular element for three-dimensional fracture mechanics analysis. Engineering Analysis with Boundary Elements, 20(4), pp. 275-285, 1997.

http://dx.doi.org/10.1016/S0955-7997(97)00070-2

[14] Mi, Y. \& Aliabadi, M.H., Discontinuous crack-tip elements: application to 3D boundary element method. International Journal of Fracture, 67(3), pp. R67-R71, 1994. http://dx.doi.org/10.1007/BF00016267

[15] Benthem, J.P., State of stress at the vertex of a quarter-infinite crack in a half-space. International Journal of Solids and Structures, 13(5), pp. 479-492, 1977. http://dx.doi.org/10.1016/0020-7683(77)90042-7

[16] Bažant, Z.P. \& Luis F.E., Surface singularity and crack propagation. International Journal of Solids and Structures, 15(5) pp. 405-426, 1979. http://dx.doi.org/10.1016/0020-7683(79)90062-3

[17] Shivakumar, K.N. \& Raju, I.S., Treatment of singularities in cracked bodies. International Journal of Fracture, 45(3), 159-178, 1990. http://dx.doi.org/10.1007/BF00693347

[18] Kwon, S.W. \& Sun, C.T., Characteristics of three-dimensional stress fields in plates with a through-the-thickness crack. International Journal of Fracture, 104(3), pp. 289-314, 2000.

http://dx.doi.org/10.1023/A:1007601918058

[19] Aliha, M.R.M. \& Saghafi, H., The effects of thickness and Poisson's ratio on 3D mixedmode fracture. Engineering Fracture Mechanics, 98, pp. 15-28, 2013. http://dx.doi.org/10.1016/j.engfracmech.2012.11.003

[20] Raju, I.S. \& Newman, J.C., Three dimensional finite-element analysis of finite-thickness fracture specimens. NASA, Washington, DC, 1977.

[21] Murakami, Y. \& Hasebe, N. (eds), Stress Intensity Factors Handbook, Elsevier Science: Amsterdam/New York, 2001. 